\title{
Wissenschaftliche Originalarbeiten in deutscher Sprache - Ein Anachronismus
}

\author{
Original Articles in German Language are an Anachronism
}

Autoren

Pro: Dieter F. Braus

Kontra: Christopher Baethge

Bibliografie

DOI 10.1055/s-0028-1090152

Psychiat Prax 2009; 36:

157-159

(c) Georg Thieme Verlag KG

Stuttgart · New York .

ISSN 0303-4259

Korrespondenzadressen

Prof. Dr. Dieter F. Braus

Direktor der Klinik und Poliklinik für Psychiatrie und Psychotherapie, HSK, Dr. Horst Schmidt Klinik GmbH

Ludwig-Erhard-Straße 100

65199 Wiesbaden

\section{PD Dr. Christopher Baethge}

Leiter der Medizinisch-Wissenschaftlichen Redaktion, Deutsches Ärzteblatt

Ottostraße 12

50859 Köln

baethge@aerzteblatt.de

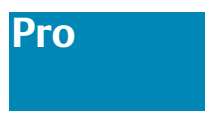

Stellt man sich die Frage, ob wissenschaftliche Originalarbeiten in deutscher Sprache heute noch zeitgemäß sind, ist es zuerst notwendig, diese Form von Veröffentlichungen zu definieren. Im Weiteren geht es darum den Kontext, also das Fachgebiet und dessen Theoriegebäude zu klären, in dem solche Arbeiten erscheinen. Und dann gilt es, die heutige Zeit und ihren Umgang mit dem globalen Wissenspool zu betrachten. Daraus können dann die Frage nach dem Anachronismus beantwortet und mögliche Perspektiven aufgezeigt werden.

\section{Originalarbeiten in der Psychiatrie}

Die Psychiatrie als medizinische Disziplin zählt heute zu den Biowissenschaften und gehört damit zu den Fachgebieten, in denen neue Forschungsergebnisse als Originalarbeiten in Fachzeitschriften vom Fachpublikum wahrgenommen werden. Diese müssen formalen und inhaltlichen Kriterien genügen, nachdem die Arbeiten von Fachgutachtern auf die wissenschaftliche Güte, ethische Grundsätze und Originalität überprüft wurden. Die Ergebnisse werden dabei in das „Theoriegebäude“ der Hirn(fehl)funktion eingeordnet. Dieses ist vom „Theoriegebäude“ der Geist-Seele-Funktion abzugrenzen, das von den Geisteswissenschaften gespeist wird. Bei den weiteren Ausführungen sollen Veröffentlichungen aus dem letztgenannten Bereich nicht beleuchtet werden.

\section{Die heutige Zeit: Globalisierung auch im Umgang mit Wissen}

Nicht nur im Handel auch in der Medizin befinden wir uns in einem Globalisierungsprozess, der sich in effizienterer Grundlagenforschung und in rascherem, nutzerfreundlichen Wissenstransfer äußert. Gleichzeitig haben sich in den Biowissenschaften mit der Molekularbiologie sowie den bildgebenden Verfahren neue, technisch aufwendige Möglichkeiten ergeben [1], die heute nur in interdisziplinären Gruppen und multizentrischen Studiendesigns erfolgreich und bezahlbar nutzbar gemacht werden können. Unter dem Einfluss der Naturwissenschaften wird die Theoriebildung über die Funktion des Gehirns ständig verfeinert, wobei aber feststellbar ist, dass zentrale Wissenslücken unbearbeitet bleiben, wo mangels Profiterwartung keine Industriefinanzierung besteht. Dieser (teilweise auch gefährliche) Globalisierungsprozess führt zu einem rasant wachsenden globalen Wissenspool in englischer Sprache. Der Zugang und die geschickte Nutzung dieser Quellen sind für den Erfolg einer Fachdisziplin heute unabdingbar.

\section{Psychiatrisches Fachgebiet im Wandel}

Die Fortschritte in den Biowissenschaften haben inzwischen auch die Psychiatrie erreicht, ein Fachgebiet, das Anfang des 20. Jahrhunderts in der frühen Hirnforschung verwurzelt war und das sich aufgrund fehlender methodischer Möglichkeiten in der Mitte des 20. Jahrhunderts vorübergehend stark mit den Geisteswissenschaften verbunden fühlte. Seit den 1990er-Jahren wurde der Fokus der Erforschung psychischer Störungen von der differenzierten Beschreibung der psychopathologischen Krankheitsphänomene oder sozialpsychiatrischen Fragestellungen hin zum Verständnis der neurobiologischen Basis psychischer Erkrankungen verlagert [2]. Die sehr kostengünstige „Papier-und-Bleistift-Forschung“ ist heute nicht mehr möglich, lokale Besonderheiten wie die Sprache für die Psychopathologie haben an Bedeutung verloren und die Beschreibung von Versorgungsstrukturen macht nur noch im internationalen Vergleich Sinn.

Wie in den Naturwissenschaften unterliegt nun auch die biowissenschaftliche Forschung in der Psychiatrie einem globalen Wettbewerb um die Forschungsmittel. Es geht heute in der klinischen Forschung z.B. darum, den Einfluss genetischer 
Faktoren auf die Pathophysiologie zu verstehen oder auf der Systemebene unter Einsatz bildgebender Verfahren Netzwerkmodelle als Grundlage für Hirnfehlfunktion auf ihre Nutzbarkeit für die Differenzialdiagnose sowie die Differentialtherapie zu überprüfen. Hierfür sind neben aufwendiger apparativer Grundausstattung und hohem methodischen Know-how auch ständige große finanzielle Ressourcen unabdingbar. Die dafür beantragten Forschungsmittel werden - neben den Interessen des Geldgebers und der Originalität des Projektes - vor allem danach verteilt, in welchen Zeitschriften mit welchem Impact-Faktor die Antragsteller schon publiziert haben. Zeitschriften mit hohem Impact-Faktor sind ausnahmslos englischsprachig, sie liegen bis zum 50-fachen über dem deutscher Journale. Ohne den Sinn dieser Faktoren hier diskutieren zu wollen, muss konstatiert werden, dass kein Zurück aus dieser Entwicklung absehbar ist.

\section{Ansprüche an Publikationen haben sich verändert}

Die methodischen Ansprüche an die psychiatrische Forschung, aber auch an die Ausbildung des Nachwuchses steigen stetig [3]. Der Peer-review-Prozess in internationalen Journalen mit hohem Impact-Faktor ist sehr effizient und hat eine hohe fachliche Qualität, da englischsprachige Zeitungen auf eine globale Gutachterschar zurückgreifen können und sich gleichzeitig die Editoren konstruktiver Kritik stellen [4-6]. Eine große Vielfalt von zum Teil hochspezialisierten englischsprachigen Fachzeitschriften ist verfügbar. Gerade junge Autoren können durch die kritischen Einlassungen ihre eingereichten Manuskripte nachhaltig verbessern und für ihre weiteren Forschungsaktivitäten profitieren. Deutschsprachige Zeitungen können aufgrund der geringeren Leserschaft weder im Impact-Faktor noch im Review-Prozess wirklich mithalten. Im globalen Wettbewerb besteht die Gefahr, dass Ergebnisse und Schlussfolgerungen in deutschsprachigen Zeitschriften eingereicht werden, die in höherrangigen Journalen schon abgelehnt wurden oder die dort nicht einmal in den Review-Prozess kamen. Kommt es zur Veröffentlichung wird häufig unnötige Redundanz gefördert [7] oder im ungünstigen Fall könnten dadurch sogar Metaanalysen, die zu Behandlungsleitlinien führen, verfälscht werden.

\section{Fazit und Perspektiven}

Deutschsprachige Originalarbeiten in der Psychiatrie sind ein Anachronismus. Originalarbeiten in dem hier skizzierten Sinne schaffen nach einem rigorosen Review-Prozess neues Wissen, das rasch allen Arbeitsgruppen in der Welt in der Wissenschaftssprache Englisch zugänglich gemacht werden muss, um die aufwendigen, weltweit laufenden Forschungsaktivitäten leichter zu fokussieren, Wissenslücken zu schließen und neue Erkenntnisse in die Gesundheitsversorgung fließen zu lassen. Der zeitliche Aufwand zum Verfassen einer Originalarbeit ist für die Autoren in jeder Sprache der Gleiche, der eigene Profit für das Weiterkommen und der Vorteil für die Forschungsgemeinschaft sind für englischsprachige Veröffentlichungen signifikant größer. Mangelndes Interesse am globalen englischsprachigen Wissenspool und nur profitorientierte Forschung sind gefährlich. Dies bedeutet jedoch nicht, dass deutschsprachige Zeitschriften anachronistisch sind. In der Praxis tätige Ärztinnen und Ärzte benötigen [8] beispielsweise zeitnah 1. gut aufbereitete, ins Deutsche übersetzte Übersichtsarbeiten von renommierten Forschern, 2. Fallberichte, die eingebettet sind in das aktuelle pathophysiologische Wissen, 3. Kurzzusammenfassungen von Originalarbeiten mit kritischer Bewertung der Ergebnisse und Einordnung in die Praxis, 4. hochwertige zertifizierte Weiterbildungsartikel oder auch 5. Pro- und Kontra-Debatten, wie diesen Artikel in deutscher Sprache, die zum Nachdenken anregen können. Und sie erfordern gute, von Interessengruppen unabhängige Lotsen in den Redaktionen, die im Dschungel der Veröffentlichungen die relevanten Originalarbeiten von den Irrelevanten trennen. Nicht zuletzt aber braucht der Leser Zeit und wirkliches Interesse, dann auch das Relevante im Original oder in der Bearbeitung zu lesen und in den Versorgungsalltag zu integrieren.

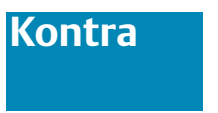

Es ist ein großes Glück für mich, dass die Psychiatrische Praxis die Debatte über das Zeitgemäße des Deutschen als Wissenschaftssprache auf Deutsch führen lässt. Was ich schreibe, bedeutet auf diese Weise auch, was ich meine. Ebenso verstellen mir keine Verständnisschwierigkeiten die Lektüre des Beitrags von Professor Braus. Auf Englisch wäre das anders: Selbst deutsche Wissenschaftler, die lange im englischsprachigen Ausland gelebt haben, vermögen sich nicht die Artikulationsfähigkeit von Muttersprachlern anzueignen; vermutlich beherrschen nur wenige deutsche Autoren und Leser die ganze Bandbreite englischer Ausdrucksmöglichkeiten und rhetorischer Finessen. Es hat sich auch empirisch gezeigt, dass Akademiker Texte in ihrer Muttersprache besser verstehen oder behalten konnten als auf Englisch $[9,10]$. Dieser Hinweis auf eine nach wie vor wirksame Sprachbarriere sei erlaubt vor der Beantwortung der Frage, ob wissenschaftliche Originalarbeiten - hier verstanden als die Publikation neuer Forschungsergebnisse - auf Deutsch ein Anachronismus sind.

Mangelnde sprachliche Raffinesse wiegt je nach Art der Originalarbeit unterschiedlich schwer, und dies ist einer der Gründe, warum die vorliegende Frage nuancierte Antworten erfordert. Je standardisierter eine Untersuchung ist, desto leichter lässt sie sich auf Englisch darstellen: Dies gilt zum Beispiel für die methodisch weitgehend vereinheitlichten Medikamentenstudien in unserem Fach. Wie sieht es aber etwa bei der Versorgungsforschung aus, einem Wissenschaftszweig, der definitionsgemäß an ein nationales medizinisches System - und oft auch an eine eigene Terminologie - gebunden ist? Während die Ergebnisse von Medikamenten- und Bildgebungsstudien oder von genetischen Forschungsprojekten aus Deutschland auch in anderen Teilen der Welt Geltung beanspruchen können und von daher in englischen Zeitschriften gut repräsentiert sind, stoßen Versorgungsforscher auf große - und in ihrem Forschungsgegenstand begründete - Schwierigkeiten, ihre Resultate international zu publizieren, weil in anderen Ländern andere Versorgungsstrukturen bestehen. Doch auch diese Ergebnisse sind wissenschaftlich und gesellschaftlich bedeutsam. Andere Disziplinen publizieren ihre Forschungsresultate ebenfalls nur oder überwiegend auf Deutsch, etwa die Medizingeschichte oder -ethik.

Grundlegende, konzeptionelle oder philosophische Arbeiten können durchaus Originalarbeiten sein, werden aber gerade wegen ihrer sprachlichen Komplexität unter normalen Umständen nur in der Muttersprache des Autors abgefasst. Es fällt schwer sich vorzustellen, dass Karl Jaspers seine Allgemeine Psychopathologie ohne Bedeutungsverlust auf Englisch hätte schreiben können. Aktuelle Beispiele aus dem Bereich der Psychopathologie wären Arbeiten von Werner Janzarik oder Thomas Fuchs, die ebenfalls auf Deutsch erschienen sind (z. B.: $[11,12]$ ).

Diese Fälle zeigen, dass wir auf unsere eigene Sprache als wissenschaftliches Verständigungsmittel nicht gänzlich verzichten sollten - vor allem, wenn wir die Psychiatrie, aber auch die Me- 
dizin insgesamt, nicht ausschließlich als ein naturwissenschaftliches Fach ansehen, sondern als eine methodenpluralistische Disziplin. Vielleicht kann als Faustregel gelten, dass zwar jeder Text in der Muttersprache besser ist als in englischer Übersetzung, dieser Zusammenhang für die geisteswissenschaftlichen Teildisziplinen aber besonders gravierend ist. Hierbei ist noch nicht einmal die Debatte berücksichtigt, ob eine Sprache nicht auch das Denken beeinflusst und daher der Verzicht auf sie eine primäre gedankliche Einengung bedeutet („Sapir-WhorfHypothese“).

Trotz der Dominanz der großen allgemeinen und fachspezifischen englischsprachigen Journale ist Deutsch weiterhin Sprache von Originalarbeiten: Die Deutsche Zentralbibliothek für Medizin führt unter ihren 6800 Periodika rund 1200 deutschsprachige Zeitschriften $[13,14]$, von denen viele nicht nur Fortbildungsartikel veröffentlichen, sondern auch Originalbeiträge - wie die Psychiatrische Praxis. Weltweit nimmt der Anteil nicht englischsprachiger Zeitschriften sogar zu [13,14]. Auf Deutsch erscheinende wissenschaftliche Arbeiten werden in der Öffentlichkeit auch durchaus wahrgenommen: Zwar hat - um nur ein Beispiel zu nennen - die FAZ 2006 und 2007 einer eigenen Zählung zufolge über viele Studien aus JAMA oder dem BMJ (je $\mathrm{n}=34$ ) berichtet, die Redakteure referierten aber durchaus auch wissenschaftliche Arbeiten aus deutschsprachigen Zeitschriften, etwa aus dem Deutschen Ärzteblatt $(\mathrm{n}=15)$ oder der DMW $(\mathrm{n}=11)$.

Ideal wäre beides: der gleiche Artikel in je einer deutschen und einer englischen Version. Das Deutsche Ärzteblatt hat diese Konsequenz gezogen: Es übersetzt jeden wissenschaftlichen Beitrag auf Englisch und publiziert ihn in seiner open access InternetZeitschrift Deutsches Ärzteblatt International (www.aerzteblattin ternational.de) $[13,14]$ Beispiele: $[15,16]$. Damit ist sowohl einer internationalen Leserschaft gedient als auch der des Deutschen Ärzteblattes. Nicht zuletzt finden die Artikel unserer Autoren so international Verbreitung, obwohl diese weiterhin auf Deutsch schreiben können. Auf dem Gebiet der Chemie ist mit der Angewandten Chemie sogar eines der weltweit führenden Journale zweisprachig. Allerdings bedeutet eine zweisprachige Ausgabe eine enorme logistische, personelle und finanzielle Aufgabe, die viele kleinere Zeitschriften nicht schultern können.

Was folgt? Deutsch sollte nicht nur eine Sprache von Originalarbeiten sein, es ist es nach wie vor. Die Frage, ob wissenschaftliche Originalarbeiten auf Deutsch erscheinen, wird auch nicht von Editorialisten entschieden, sondern von den Wissenschaftlern. Sie sollten dort ihre Manuskripte einreichen können, wo sie ein Publikum finden, das ihre Arbeit zu würdigen weiß. Dabei ist es natürlich für die globale Wissenschaftlergemeinde immer ein Gewinn und in einigen Bereichen zwingend nötig, Studienergebnisse auf Englisch für alle einsehbar vorzulegen. Wichtig wäre aber, dass die Forschungsbürokratie in Deutschland nicht solche Wissenschaftler diskriminiert, die auf Deutsch veröffentlichen wollen oder müssen. Eine solche Benachteiligung erfolgt schon durch die Fokussierung auf den Impact-Faktor als alleini- gen Bewertungsmaßstab. Der Impact-Factor bevorzugt englischsprachige Zeitschriften insofern, als Spitzenwerte nur durch eine Akkumulation von Zitaten in vielen Journalen entstehen können, und ausschließlich der englische Sprachraum - anders als der französische, deutsche oder spanische - eine solche Vielzahl von Zeitschriften bietet [17]. Hier ist noch nicht einmal die Problematik berücksichtigt, dass einige anglophone Zeitschriften nicht englischsprachige Literaturangaben unterbinden [18].

In der Forderung, deutsche Autoren müssten alles auf Englisch zu publizieren, also auch alles auf Englisch auszudrücken in der Lage sein, scheint sich mir eine Unterschätzung der Bedeutung der Muttersprache für weite Bereiche der Wissenschaft zu zeigen. Es wäre schön, wenn die mit hiesigem Geld finanzierten Wissenschaftsministerien, Forschungsförderorganisationen und auch Dekanate sich etwas selbstbewusster zu Deutsch als einer wichtigen Wissenschaftssprache bekennen würden.

\section{Literatur}

1 Braus DF. A picture of the mind. Nuclear magnetic resonance tomography gives the first view of the somatic basis of psychic processes. Med Monatsschr Pharm 2003; 26: 45-51

2 Krishnan V, Nestler EJ. The molecular neurobiology of depression. Nature 2008; 455: 894-902

3 Roffman JL, Simon AB, Prasad KM et al. Neuroscience in psychiatry training: how much do residents need to know? Am J Psychiatry 2006; 163: 919-926

4 Peer review and fraud. Nature 2006; 444: 971-972

5 Alberts B, Hanson B, Kelner KL. Reviewing peer review. Science 2008; 321: 15

6 Jefferson T, Wager E, Davidoff $F$. Measuring the quality of editorial peer review. JAMA 2002; 287: 2786-2790

7 Mojon-Azzi SM, Jiang X, Wagner U et al. Journals: redundant publications are bad news. Nature 2003; 421: 209

8 Rochon PA, Bero LA, Bay AM et al. Comparison of review articles published in peer-reviewed and throwaway journals. JAMA 2002; 287: 2853-2856

9 Gulbrandsen P, Schroeder TV, Milerad J et al. Paper or Screen, Mother Tongue or English: Which is Better? JAMA 2002; 287: 2851-2853

10 Airey J, Linder C. Language and the Experience of Learning University Physics in Sweden. Eur J Phys 2006; 27: 553-560

11 Fuchs T. Ökologie des Gehirns. Eine systemische Sichtweise für Psychiatrie und Psychotherapie. Nervenarzt 2005; 76: 1-10

12 Janzarik W. Wollen und Wille. Nervenarzt 2008; 79: 567-570

13 Baethge C. Die Sprachen der Medizin. Dtsch Arztebl 2008; 105: 37-40

14 Baethge $C$. The Languages of Medicine. Dtsch Ärztebl Int 2008; 105: $37-40$

15 Lulé D, Häcker S, Ludolph A et al. Depression und Lebensqualität bei Patienten mit amyotropher Lateralsklerose. Dtsch Ärztebl 2008; 105: 397-403

16 Lulé D, Häcker S, Ludolph A et al. Depression and Quality of Life in Patients with Amyotrophic Lateral Sclerosis. Dtsch Ärztebl Int 2008; 105: 397-403

17 Winkmann G, Schlutius S, Schweim HG. Publikationssprache der Impact-Faktor-Zeitschriften und medizinischer Literaturdatenbanken. Dtsch Med Wochenschr 2002; 127: 131-137

18 Lemmer B, Middeke M. Geschichte einer wissenschaftlichen Publikation - nur noch englische Zitate erwünscht. Dtsch Med Wochenschr 2008; $133: 2699$ 